OPEN ACCESS

Edited by:

Mauro Serafini,

University of Teramo, Italy

Reviewed by:

Naomi Kakoschke,

Commonwealth Scientific and

Industrial Research Organisation

(CSIRO), Australia

Marie-Pierre St-Onge,

Columbia University, United States

${ }^{*}$ Correspondence: MoezAllslam Ezzat Faris mfaris@sharjah.ac.ae,

moezfaris@hotmail.com

tThese authors have contributed equally to this work

Specialty section:

This article was submitted to Nutritional Epidemiology,

a section of the journal

Frontiers in Nutrition

Received: 31 October 2021 Accepted: 10 December 2021

Published: 19 January 2022

Citation:

Naja F, Hasan H, Khadem SH, Buanq MA, Al-Mulla HK, Aljassmi AK and Faris ME (2022) Adherence to the Mediterranean Diet and Its Association

With Sleep Quality and Chronotype Among Youth: A Cross-Sectional

Study. Front. Nutr. 8:805955. doi: 10.3389/fnut.2021.805955

\section{Adherence to the Mediterranean Diet and Its Association With Sleep Quality and Chronotype Among Youth: A Cross-Sectional Study}

\author{
Farah Naja ${ }^{\dagger}$, Hayder Hasan ${ }^{\dagger}$, Safiya Hassan Khadem, Maryam Ahmed Buanq, \\ Haya Khalid Al-Mulla, Aysha Khalifa Aljassmi and MoezAllslam Ezzat Faris*

\begin{abstract}
Department of Clinical Nutrition and Dietetics, College of Health Sciences, Research Institute for Medical and Health
\end{abstract} \\ Sciences (RIMHS), University of Sharjah, Sharjah, United Arab Emirates
}

Background: Evidence indicates that many university students have poor adherence to a healthy diet accompanied by unhealthy lifestyle behaviors. Chrono-nutrition is an emerging field of research that examines the pattern of optimum daily activity in relation to the human's dietary patterns, and their reflections of variable health indicators such as sleep quality. However, there is a scarcity of research that examines the relationship between adherence to the healthy eating pattern, like the Mediterranean diet (MD), with sleep quality and chronotype among university students.

Methods: A cross-sectional study was conducted, and convenience sampling was used. Participants were assessed for adherence to the MD using the Mediterranean Diet Quality Index (KIDMED), for sleep quality using the Pittsburgh Sleep Quality Index (PSQI), and for chronotype using the Morningness-Eveningness questionnaire (MEQ).

Results: The study included 503 university students, most of them (81.5\%) were females. Only 15.1 and 16.9\% reported morningness chronotype and good sleep quality, respectively. About half of the students showed medium and high adherence to the KIMED. In-depth analysis revealed that students with good adherence to the MD were more likely to have a good sleep quality $(\mathrm{OR}=0.35 ; 95 \% \mathrm{Cl}$ : $0.21-0.59 ; P<0.001)$ even after adjustment for age and sex $(\mathrm{OR}=0.36$; 95\% Cl: $0.21-0.62 ; P<0.001)$. The regression analysis also showed that those with good adherence to the MD had a significant association with better subjective sleep quality, less sleep latency, sleep disturbance, and daytime dysfunction even after adjustment for age and sex. Those with morningness chronotype had about a six-fold higher chance to have good adherence to the $\mathrm{MD}(\mathrm{OR}=5.67 ; 95 \% \mathrm{Cl}: 2.86-11.26 ; P<0.001$, respectively).

Conclusions: Good adherence to the healthy diet presented in the MD among university students is associated with morningness chronotype and with improved overall sleep quality and sleep components. Long-term, controlled intervention research works are warranted for more elaboration on the impact of chronotype and dietary habits on sleep quality and other important aspects such as mental health and academic achievement.

Keywords: healthy diet adherence, chrononutrition, chronobiology, circadian and sleep physiology, sleep quality, circadian rhythm 


\section{INTRODUCTION}

The Mediterranean diet (MD), native to countries surrounding the Mediterranean basin, has been consistently associated with improved individual health $(1,2)$. The MD is characterized by increased consumption of a wide variety of local and seasonal fruits and vegetables, whole grains, moderate amounts of dairy products, plant-based protein sources, with olive oil and olives being the main source of fat. In addition, MD contains limited amounts of red meat. Within this diet, salt is partially replaced by the use of spices and herbs (3). The MD has recently been described as a lifestyle pattern that extends beyond dietary intake where regular physical activity, sharing meals with other people, and enjoying life are all part of this pattern (4). Research on MD indicates that it is rich in vitamins, fibers, probiotics, monounsaturated fatty acids (MUFA) and polyunsaturated fatty acids (PUFA), bioactive antioxidants and low glycemic foods, as well as anti-inflammatory agents; all of which contribute to impacting better lipid profile and reducing obesity and the associated inflammatory state, metabolic syndrome, type 2 diabetes mellitus (T2DM) and cardiovascular disease (CVD) (5-10). Several physiological mechanisms have been suggested to mediate the effect of the MD on health including microbiome diversity, lowering low-density lipoproteins (LDL) levels, oxidative stress, and inflammation, and an improvement in immune function and insulin sensitivity (6). In addition to these physiological mechanisms, higher adherence to the MD has been associated with lower c-reactive protein (CRP) and inflammatory cytokines secretion, including interleukin-1 (IL-1), tumor necrosis factor- $\alpha$, and IL-6 (11). Increased levels of these inflammatory markers have been linked with poor sleep quality among different groups of people $(12,13)$.

Sleep, an important dimension, and determinant of lifestyle is a complex homeostatic process that involves multiple processes, including sleep-wake homeostasis, circadian cycle, and human dietary and lifestyle behaviors (14). Adequate sleep quality plays a pivotal role in health and exerts a positive influence on psychomotor performance, neurocognitive functioning, and physical health (15). Globally, reduced sleep quality is increasing at an alarming rate in the current era (16), mostly due to the excessive use of the blue screen especially at night times (17). Poor sleep quality has been associated with increased risks for obesity, T2DM, and CVD (18).

Related to sleep is the chronotype, defined as the expression of an individual's diurnal preferences in experiencing various psychological and biological traits including the wakefulness and timing of sleep (19). Individuals who sleep and wake up early and perform daily activities optimally during the morning hours are classified as having a morningness preference or are known as morning-type. In contrast, those who sleep and wake up late, ideally perform their daily activities in the evening hours are classified as having an eveningness preference or are recognized as evening-type. The intermediate type refers to individuals who can be either morning or evening type. The chronotype is a characteristic of the circadian timing system that presents differences between sexes and is affected by geographical factors such as latitude $(20,21)$. Chronotype has been shown to modulate sleep quality, sleep duration, and social jet lag in shift-workers (22). This notion supports the relationship between chronotype and sleep quality, and strengthens the hypothesis that sleep quality is better in morning types than evening types due to the social jetlag often experienced by the people with evening chronotype $(23,24)$.

A growing body of evidence signifies the relationship between diet and sleep, with several foods and food components that have been found to improve sleep duration and quality. Among these, tryptophan, and foods containing tryptophan, melatonin, and phytonutrients were looked at as sleep-promoting foods linked to improved sleep outcomes $(25,26)$, with those foods triggering the synthesis of serotonin and melatonin counted among the most helpful in promoting sleep (27). Further, dietary factors can profoundly impact the inflammatory status and hormones that can either directly or indirectly contribute to poor sleep quality, with variable effects for the major nutritional factors such as lipids, carbohydrates, and vitamins on sleep and sleep disorders (28).

While the MD and adequate sleep quality are important determinants of health throughout the lifecycle, they are of utmost importance among youth and adolescents. Youth and young adulthood is a period between 15 and 25 years of age, characterized by dramatic psychological and physiological changes, such as increased levels of autonomy in decisionmaking and increased self-development and identity (29). People at that age tend to have a nutrient-poor diet, often accompanied by high consumption of sugar-sweetened beverages and other energy-dense fast food (30). Among youth and adolescents, college students are particularly vulnerable to poor dietary habits because they have to adapt to new lifestyle changes associated with increased independence, timeconsuming and hectic schedules, and high academic demands and responsibilities (31). These factors together were associated with increased consumption of caffeine, energy drinks $(32,33)$, and fast foods (34) by university students. University students' diets were shown to be low in vegetables and fruits, and high in sodium, carbohydrates, fats, and, making them energy-dense yet nutrient-poor options (35). University students in the United Arab Emirates (UAE) have been found to eat unplanned meals consisting primarily of unhealthy, nutrient-poor, and energydense foods (36). Furthermore, university students in the UAE have been observed to consume a lot of soft drinks and fast food, as well as skipping meals $(34,37)$. The negative health implication of such poor dietary habits among university and college students are further exacerbated by suboptimal sleep quality. Studies have shown that this population group is at high risk for sleep disorders (38). A review of the literature revealed that $55 \%$ of college students in the Gulf Cooperation Council (GCC) have irregular sleep schedules and get $<7 \mathrm{~h}$ of sleep per night (39). While the relationship between adherence to the MD and sleep quality has been the subject of intensive research, little is known about the association between adherence to the MD and chronotype among university students. Thus, this study aimed primarily to (1) Examine the degree of adherence to the MD, sleep quality, and chronotype among youth, (2) Investigate the relationship between adherence to the MD on sleep quality, and 
(3) Explore the relationship between chronotype and adherence to the $\mathrm{MD}$, and finally, (4) to explore whether there is an association between sleep quality and chronotype.

\section{METHODS}

\section{Study Design and Participants}

To achieve the study objectives, a cross-sectional survey was conducted among students at the University of Sharjah (UoS), Sharjah, UAE. The UoS is a national university that has a diverse study body, coming from various ethnicities and backgrounds. Data collection was carried out between January and May 2021, using a convenience sampling approach. An email inviting students to take part in the study was sent by the central administration to all students. Students, both males, and females, aged 18 years and above and registered at the university during the time of data collection were eligible to participate in this study. The protocol of this study was designed and implemented in line with the guidelines specified by the Declaration of Helsinki. The survey's protocol and data collection instrument were reviewed and approved by the Research Ethics Committee at the UoS (REC-21-03-03-09-S). Before the start of data collection, participants were presented with a brief introduction about the study, its objectives, and procedure, and were asked to provide a signed electronic informed consent, in case they agreed to participate. Participants were informed that their participation is anonymous and completely voluntary in that they can choose or not to answer any particular question and that they are free to withdraw from the study at any time. Participation was completely voluntary, with no monetary or non-monetary incentives were given to the study participants.

Sample size calculations showed that 479 students are needed to estimate a $13.3 \%$ prevalence of good adherence to the MD with a power of $80 \%$ and an error margin of $3 \%(40)$. The $13.3 \%$ prevalence estimate used in the sample size calculations in this study was based on the results of a previous investigation of adherence levels to the MD among adolescents, conducted in Lebanon, a neighboring country of the Middle East and North African Region (41). The sample was representative of the students population of the UoS as it included the same proportion of males to females (1:4) at the UoS, the majority of the students were UAE nationals and/or GCC citizens. Likewise, the medical and health sciences colleges were 4 out of 14 colleges, which is reflected by the number of participants from different college majors $(29.6 \%$ medical and health sciences and $70.4 \%$ from other colleges).

\section{Data Collection Tool}

For the data collection, a multicomponent, self-administrated online questionnaire was designed using Google Forms in both the English and Arabic languages. The link for the questionnaire was included within the body of the emails distributed to the participants. A panel of experts, consisting of a clinical nutritionist, a nutrition epidemiologist, and a public health scientist, developed the questionnaire choosing the scales for each of the concepts under study and ensuring its content validity. The questionnaire was first developed in the English language, later translated into Arabic, and backtranslated into English. The original and back-translated versions of the questionnaire were compared for consistency and to ensure parallel-form reliability. Following the development of the questionnaire, a pilot test was conducted on 15 students to ensure cultural and context adaptability. The results of the pilot test were not included in the data for this study.

The questionnaire consisted of four main sections that addressed (1) sociodemographic characteristics, 2) adherence to the Mediterranean diet, (3) sleep quality, and (4) sleep chronotype. Except for the first section, the questionnaire referred to the month prior to the participation in the study. The average duration for the completion of the questionnaire was $10 \mathrm{~min}$.

Section Introduction included questions to provide the following information about the participants: age (in years), sex (male; female), nationality (GCC-; non-GCC), marital status (single, married), income (low: $<5,000$ United Arab Emirates dirham [AED]; medium: 5,000-10,000 AED and high: $>10,000$ AED), smoking status (non-smoker, including past smoker, smoker) years spent at the university ( $<4, \geq 4$ years), cumulative grade point average (CGPA) $(\leq 3,>3)$, and major of studies at the university (health-related, non-health-related). In addition, participants were asked to report their weight $(\mathrm{kg})$ and height $(\mathrm{cm})$. Accordingly, the participant's body mass index (BMI) was calculated as weight $(\mathrm{kg}) /[\text { height }(\mathrm{m})]^{2}$.

The second section of the questionnaire examined adherence to the MD. For that purpose, The Mediterranean Diet Quality Index (KIDMED) was used. A systematic review of tools used showed that the KIDMED is the most widely used index to assess adherence to the MD among youth (42). The reliability of this index was found at $r=0.60$ among college students (43). This index, first developed by Serra-Majem et al. (44), addressed food habits specific to the Mediterranean region. One of the important advantages of the KIDMED is that it can be self-administered as well as filled by an interviewer. The KIDMED consisted of 16 questions to which the answers could be either yes or no. Twelve of those questions were related to positive food habits and were related to the consumption of 1-fruits or fruit juice (daily), 2second serving of fruit (daily), fresh or cooked vegetables (daily), fresh or cooked vegetables (more than once per day) fish (at least 2-3 times per week), legumes (more than once per week), pasta or rice ( $\geq 5$ times per week), nuts (at least 2-3 times per week), use of olive oil at home, dairy products ( 2 yogurts and/or $40 \mathrm{~g}$ cheese daily), including cereals or grains for breakfast, and dairy for breakfast. For these positive food habits, the participants obtained 1 point for answering yes and 0 points for answering no, toward their KIDMED score. The remaining four questions, addressed negative food habits, consisting of skipping breakfast, including commercially baked goods or pastries for breakfast, sweets, and candy several times a day, and going to a fastfood restaurant more than once a week. For each of these four questions, the participant received a -1 if his/her answer was Yes and 0 if his/her answer was No. The sum of the points for all the 16 questions was calculated into a KIDMED score, ranging from $(-3)$ to $(+12)$, with higher scores indicating better adherence. Accordingly participating were grouped into three 
categories: good adherence ( $\geq 8$ ), average (between 4 and 7 ), and poor $(\leq 3)(44)$.

The third section of the questionnaire addressed the sleep quality among study participants (45). The Arabic version of the Pittsburgh Sleep Quality Index (PSQI) was used. The Arabic version of the PSQI was previously investigated for its validity and reliability and was found to be appropriate for use among Arabic-speaking natives (46). The PSQI is a selfrated questionnaire consisting of 19 questions addressing seven components/domains of sleep quality including subjective sleep quality, sleep latency, sleep duration, habitual sleep efficiency, sleep disturbances, use of sleeping medication, and daytime dysfunction. For each of those components, participants were assigned a score of " $0-3$," whereby " 0 " indicates good, and " 3 " indicates "worst'. An overall PSQI score for sleep quality is generated as the sum of scores for the 7 components. As such, the overall PSQI ranged between 0 and 21 with higher scores indicating worse sleep quality. Using the overall PSQI score, participants were grouped as either having good $(\leq 5)$ or poor $(>5)$ sleep quality.

The last section of the questionnaire examined the participants' sleep chronotype (diurnal preferences) using the Morningness-Eveningness Questionnaire (MEQ), first developed by Horne and Östberg (47). A review of instruments used to evaluate chronotype indicated that the MEQ has been the gold standard addressing both sleep-wake information and appetite/exercise preferences while taking into account both psychological and behavioral factors when evaluating chronotypes (48). The MEQ consisted of 19 questions addressing personal daily sleep-wake habits and the times of day of preference of certain activities. Most of these questions are preferential whereby participants are asked to indicate when, for example, he/she would prefer to wake up or start sleep, rather than when he/she does. The MEQ questions are multiple-choice in nature, with each answer being assigned a specific value. The sum of these values results in an overall score ranging from 16 to 86 which can be categorized as follows: Definitely morning type 70-86, Moderately morning type 59-69, Neither type 42-58, Moderately evening type 31-41, Definitely evening type 16-30. The validity of the Arabic version of the MEQ was previously established and the results indicated that this version of the questionnaire provided valid and reliable measures of sleep chronotype (49).

\section{Data Analysis}

Data analyses for this study were carried out using the Statistical Package for Social Sciences (SPSS) for Mac HD version 23 (SPSS Inc., Chicago, IL, USA). Participants' characteristics were described as mean (standard deviation), and frequency and proportions, for continuous and categorical variables, respectively. The characteristics were presented for the overall sample and by adherence to the MD. For the latter, participants were grouped, as per the KIDMED index, into "low", "medium", and "high" adherence. Comparisons of participants' characteristics between the poor and high adherence to the KIDMED were carried out using independent student's $t$-test for continuous variable and Chi-square tests for categorical variables. In order to investigate the effect of adherence to the $\mathrm{MD}$ on sleep quality, simple and multiple logistic regressions analyses were carried out, with the adherence to the MD (high vs. low) as the independent variable and sleep quality indicators as dependent variables. Sleep quality indicators consisted of the overall PSQI and its seven components. The effect of sleep chronotype on adherence to the MD was also examined using simple and multiple logistic regression analyses. For these

TABLE 1 | Sociodemographic characteristics of the participants $(n=503)$.

\begin{tabular}{|c|c|c|c|c|}
\hline \multirow[t]{2}{*}{ Variables } & \multirow[t]{2}{*}{$n(\%)$} & \multicolumn{2}{|c|}{$\begin{array}{l}\text { Adherence to } \\
\text { Mediterranean diet } \\
\text { (KIDMED) }\end{array}$} & \multirow[t]{2}{*}{$P$-value } \\
\hline & & $\begin{array}{c}\text { Low } \\
n=231 \\
(45.9 \%)\end{array}$ & $\begin{array}{c}\text { High }^{\star \star} \\
n= \\
272(54.1 \%)\end{array}$ & \\
\hline Age $(\text { Years })^{*}$ & $22.11(4.2)$ & $21.58(3.5)$ & $22.57(4.67)$ & 0.008 \\
\hline \multicolumn{5}{|l|}{ Sex } \\
\hline Female & $410(81.5)$ & 180(43.9) & 230(56.1) & \multirow[t]{2}{*}{0.056} \\
\hline Male & $93(18.5)$ & $51(54.8)$ & $42(45.2)$ & \\
\hline \multicolumn{5}{|l|}{ Nationality } \\
\hline GCC & $444(88.3)$ & 207(46.6) & $237(53.4)$ & \multirow[t]{2}{*}{0.38} \\
\hline $\begin{array}{l}\text { Non-GCC } \\
\text { Arab/Non-Arab }\end{array}$ & $59(11.7)$ & $24(40.7)$ & $35(59.3)$ & \\
\hline \multicolumn{5}{|c|}{ Body mass index (BMI, $\mathrm{kg} / \mathrm{m}^{2}$ ) } \\
\hline $\begin{array}{l}\text { Underweight/Normal } \\
\text { weight }\end{array}$ & $339(67.4)$ & $156(46)$ & $183(54)$ & \multirow[t]{2}{*}{0.95} \\
\hline Overweight/obese & 164(32.6) & $75(45.7)$ & 54.3) & \\
\hline \multicolumn{5}{|l|}{ Marital status } \\
\hline Single & $441(87.7)$ & 206(46.7) & $235(53.3)$ & \multirow[t]{2}{*}{0.34} \\
\hline Married & $62(12.3)$ & $25(40.3)$ & $37(59.7)$ & \\
\hline \multicolumn{5}{|c|}{ Income in AED $(N=335)$} \\
\hline $\operatorname{Low}(<5,000)^{\$}$ & $259(51.5)$ & $127(49)$ & 132(51) & \multirow[t]{3}{*}{0.44} \\
\hline $\begin{array}{l}\text { Medium } \\
(5,000-10,000)^{\$}\end{array}$ & $48(9.5)$ & $20(41.7)$ & $28(58.3)$ & \\
\hline $\operatorname{High}(>10,000)^{\$}$ & $28(5.6)$ & 11(39.3) & $17(60.7)$ & \\
\hline \multicolumn{5}{|l|}{ Smoking status } \\
\hline $\begin{array}{l}\text { Non-smoker (including } \\
\text { past smokers) }\end{array}$ & $467(92.8)$ & $215(46)$ & 252(54) & \multirow[t]{2}{*}{0.85} \\
\hline Smoker & $36(7.2)$ & $16(44.4)$ & 20(55.6) & \\
\hline \multicolumn{5}{|l|}{ Years at the university } \\
\hline$<4$ years & $265(52.7)$ & $127(47.9)$ & $138(52.1)$ & \multirow[t]{2}{*}{0.34} \\
\hline$\geq 4$ years & $238(47.3)$ & 104(43.7) & 134(56.3) & \\
\hline \multicolumn{5}{|l|}{ CGPA } \\
\hline$\leq 3$ & $219(43.5)$ & $103(47)$ & $116(53)$ & \multirow[t]{2}{*}{0.66} \\
\hline$>3$ & 284 (56.5) & $128(45.1)$ & $156(54.9)$ & \\
\hline \multicolumn{5}{|l|}{ College major } \\
\hline $\begin{array}{l}\text { Medical and Health } \\
\text { Sciences }\end{array}$ & 149 (29.6) & $70(47)$ & $79(53)$ & \multirow[t]{2}{*}{0.76} \\
\hline Non-medical & 354 (70.4) & $161(45.5)$ & $193(54.5)$ & \\
\hline
\end{tabular}

"Mean (Standard Deviation, SD); BMI, Body mass index; GCC, Gulf Cooperation Council; CGPA, Cumulative Grade Point Average out of 4; BMI (in $\mathrm{kg} / \mathrm{m}^{2}$ ) according to the WHO classification: $<18.5=$ underweight, $18.5-24.9=$ Normal weight, $25-29.9$ and $\geq 30$ = Obese).

Medium and high were included as high.

$\$ 1$ United States dollar = 3.67 AED (United Arab Emirates Dirham). 
analyses, the three classes of adherence to the MD were recategorized into two categories (medium and high as one "High adherence," and "Low adherence") and considered as dependent variables, while the five chronotypes were re-categorized into three categories (eveningness, intermediate, and morningness) and entered as the independent variables. For all analyses, variables that showed significant associations with adherence to the MD in the univariate analyses were included in the multiple regression models. Results of the logistic regression analyses were presented as odds ratio (OR) and their corresponding $95 \%$ confidence interval (CI). A $p$-value $<0.05$ was considered statistically significant.

\section{RESULTS}

A total of 503 participants ( $81.5 \%$ were females) with a mean age of 22.11(4.2) years and a BMI of 23.97(2.09) $\mathrm{kg} / \mathrm{m}^{2}$ and about one third were overweight/obese $(32.6 \%)$. The majority were GCC nationals $(88.3 \%)$ and single $(87.7 \%)$, whereas about half of them had low income $(51.5 \%)$ and $52.7 \%$ were in their firstthird year of study. More than half of the students (56.5\%) had a CGPA of $>3 / 4$. The vast majority were non-smokers $(92.8 \%)$ and from non-medical and health sciences colleges (70.4\%). Only the minority had morningness chronotype and a good PSQI (15.1 and $16.9 \%$, respectively). About 54\% (272/503) of the students showed medium and high adherence to the KIMED (Table 1).

Table 1 also demonstrates the comparisons of KIDMED adherence with different sociodemographic variables. Those with medium and high adherence to KIDMED were significantly older than those with low adherence [22.57(4.67) vs. 21.58(3.5) years] and females showed more adherence compared to males $(56.1 \%$ vs. $45.2 \%, P=0.056)$.

Figure 1 illustrates the prevalence of each KIDMED component. About one-fifth of the participants were having a second fruit every day and fresh or cooked vegetables more than once a day (19.1 and 21.9\%, respectively). Additionally, more than half of the participants were consuming dairy products for breakfast $(52.9 \%)$, cereals or grains for breakfast $(63 \%)$, pasta or rice almost every day $(68.8 \%)$, and using olive oil at home $(81.3 \%)$. On the other hand, $61.2,63.4$, and $69.4 \%$ of the participants were going to a fast-food restaurant more than once a week, having commercially baked goods or pastries for breakfast, and taking sweets and candy several times every day, respectively. While three-quarters $(74.4 \%)$ of the participants were skipping breakfast.

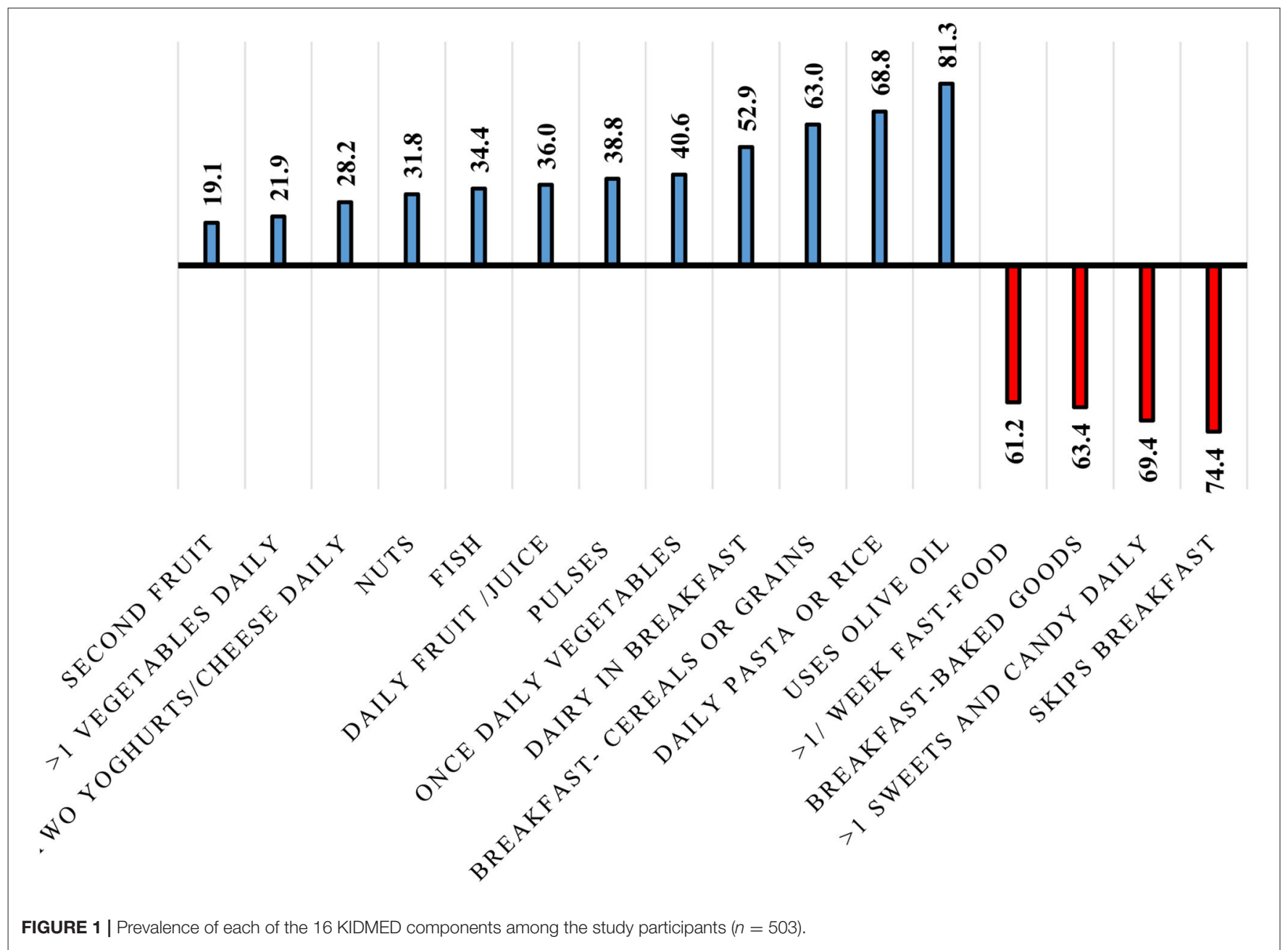


Table 2 demonstrates the association of the KIDMED with MEQ, PSQI, and PSQI components. Regarding chronotype, $80.3 \%$ of those with morningness chronotypes exhibited medium and high adherence to KIDMED compared to $39.8 \%$ for those with eveningness chronotypes $(P<0.001)$. Similarly, those with good PSQI (PSQI score $\leq 5)$ were about 3 times significantly $(P$ $<0.001)$ more adherent to the KIDMED (74.1\%) compared to those with poor PSQI (50\%). In-depth analysis of the association of the KIDMED adherence with the PSQI components showed that those with good subjective sleep quality (60.2\%), no sleep latency $(60.5 \%)$, no sleep disturbance $(57.4 \%)$, and no daytime dysfunction (60.5\%) were significantly more adherent to medium and high KIDMED.

Figure 2 depicts that students with morningness chronotype were more likely to have good PSQI compared to those eveningness chronotype ( $30.3 \%$ vs. $10.6 \%, P<0.001)$.

Table 3 shows the lack association of the overall PSQI and the KIDMED. Those with high KIDMED adherence had a significant association with subjective sleep quality $(\mathrm{B}=-0.78$; $\mathrm{OR}=0.48$; 95\% CI: 0.31-0.67; $P<0.001$ ), sleep latency ( $\mathrm{B}=-0.53$; OR $=0.58 ; 95 \%$ CI: $0.41-0.83 ; P=0.003)$, sleep disturbance $(\mathrm{B}=$ $-0.39 ; \mathrm{OR}=0.67 ; 95 \% \mathrm{CI}: 0.46-0.98 ; P=0.04)$, and daytime dysfunction $(\mathrm{B}=-0.81$; OR $=0.44 ; 95 \% \mathrm{CI}$ : $0.30-0.64 ; P<$ 0.001 ) even after adjustment for age and sex.

Table 4 demonstrates the association of the KIDMED with the MEQ. Those with morningness chronotype had about six-fold higher chance to have medium and high KIDMED adherence (unadjusted $\mathrm{OR}=6.14$; 95\%CI: 3.14-12; $P<0.001$ and adjusted $\mathrm{OR}=5.67 ; 95 \% \mathrm{CI}: 2.86-11.26 ; P<0.001$, respectively)

\section{DISCUSSION}

The current study is the first to examine the relationship between adherence to $\mathrm{MD}$, subjectively measured sleep quality using PSQI, and the chronotype among university students. Findings of the current work showed that greater adherence to the MD is associated with better overall sleep quality and sleep components.

Sleep quality is strongly affected by food quantity and quality, with dietary inflammatory potential has been proposed as one of the inflecting factors that affect sleep quality and duration (50). Providing that lower inflammatory status has been consistently associated with improved sleep quality (51, 52), the well-established low antioxidant and anti-inflammatory potential of the MD (53) may help to explain, in part, the strong positive association between the good adherence to the MD and the reported good sleep quality components found among our study sample. The relationship between sleep and inflammation is controversial and bidirectional $(12,54,55)$; that is, when the bodily inflammatory state is increased, the sleep quality is worsened, and when sleep quality is worsened, the inflammatory state becomes increased. The mechanism that elaborates such a relationship stems from the fact that inadequate sleep can increase the inflammatory response through increased cytokine secretion, including interleukin-1 (IL-1), tumor necrosis factor- $\alpha$, and IL-6; the cytokines that are classically found to be associated with sleep deprivation (12). These pro-inflammatory cytokines
TABLE 2 | Sleep quality and chronotype, overall and by the level of adherence to the MD, among study participants $(n=503)$.

\begin{tabular}{|c|c|c|c|c|}
\hline \multirow[t]{2}{*}{ Variables } & \multirow[t]{2}{*}{$n(\%)$} & \multicolumn{2}{|c|}{ KIDMED } & \multirow[t]{2}{*}{$\boldsymbol{P}$-value * } \\
\hline & & $\begin{array}{c}\text { Low } \\
n=231 \\
(45.9 \%)\end{array}$ & $\begin{array}{c}\text { Medium } \\
\text { and High } \\
n= \\
272(54.1 \%)\end{array}$ & \\
\hline \multicolumn{5}{|l|}{$\mathbf{M E Q}^{\mathrm{a}}$} \\
\hline Eveningness & 123(24.5) & $74(60.2)$ & $49(39.8)$ & $<0.001$ \\
\hline Intermediate & $304(60.4)$ & 142(46.7) & 162(53.3) & \\
\hline Morningness & $76(15.1)$ & 15(19.7) & 61(80.3) & \\
\hline \multicolumn{5}{|c|}{ Overall PSQI Score } \\
\hline Poor (>5) & 418(83.1) & $209(50)$ & $209(50)$ & $<0.001$ \\
\hline Good $(\leq 5)$ & 85(16.9) & 22(25.9) & $63(74.1)$ & \\
\hline \multicolumn{5}{|c|}{ Components of the PSQI ${ }^{\mathrm{b}}$} \\
\hline \multicolumn{5}{|c|}{ Subjective sleep quality } \\
\hline Poor & 161(32) & $95(59)$ & $66(41)$ & $<0.001$ \\
\hline Good & $342(68)$ & 136(39.8) & 206(60.2) & \\
\hline \multicolumn{5}{|c|}{ Sleep latency } \\
\hline Poor & $247(49.1)$ & 130(52.6) & $117(47.4)$ & 0.003 \\
\hline Good & 256(50.9) & 101(39.5) & 155(60.5) & \\
\hline \multicolumn{5}{|c|}{ Sleep duration } \\
\hline Poor & 107(21.3) & $57(53.3)$ & $50(46.7)$ & 0.08 \\
\hline Good & 396(78.7) & 174(73.9) & 222(56.1) & \\
\hline \multicolumn{5}{|c|}{ Sleep efficiency } \\
\hline Poor & 143(28.4) & $71(49.7)$ & $72(50.3)$ & 0.29 \\
\hline Good & $360(71.6)$ & $160(44.4)$ & 200(55.6) & \\
\hline \multicolumn{5}{|c|}{ Sleep disturbance } \\
\hline Poor & 170(33.8) & 89(52.4) & $81(47.6)$ & 0.04 \\
\hline Good & 333(66.2) & 142(42.6) & 191(57.4) & \\
\hline \multicolumn{5}{|c|}{ Use of sleep medication } \\
\hline Poor & 52(10.3) & $25(48.1)$ & $27(51.9)$ & 0.74 \\
\hline Good & 451(89.7) & $206(45.7)$ & 245(54.3) & \\
\hline \multicolumn{5}{|c|}{ Daytime dysfunction } \\
\hline Poor & 161(32) & $96(59.6)$ & $65(40.4)$ & $<0.001$ \\
\hline Good & $342(68)$ & 135(39.5) & $207(60.5)$ & \\
\hline
\end{tabular}

a'Morningness' which includes 'Definitely morning' and 'Moderately morning', Intermediate, corresponding to 'Neither morning nor evening' and Eveningness consisting of 'Definitely evening' and 'Moderately evening'.

b'good' corresponds to scores of 0 and 1; 'poor' corresponds to score 2 and 3 on each of the components.

*Using Chi-square test.

are those consistently reported to be reduced in response to the long-term exposure and adherence to the $\mathrm{MD}$, as revealed by many reports $(11,56)$.

Another underlying mechanism that explains the relationship between MD and improved sleep quality is the ability of the $\mathrm{MD}$ to increase melatonin secretion. Many fruits and vegetables are good sources of melatonin, and experimental studies reveal that consumption of these phytochemical-rich foods can improve sleep quality parameters and alleviate sleep disturbances $(26,57)$.

Further, the relationship between poor sleep quality and food intake has been consistently examined. Poor sleep quality and short sleep duration are associated with greater food intake 


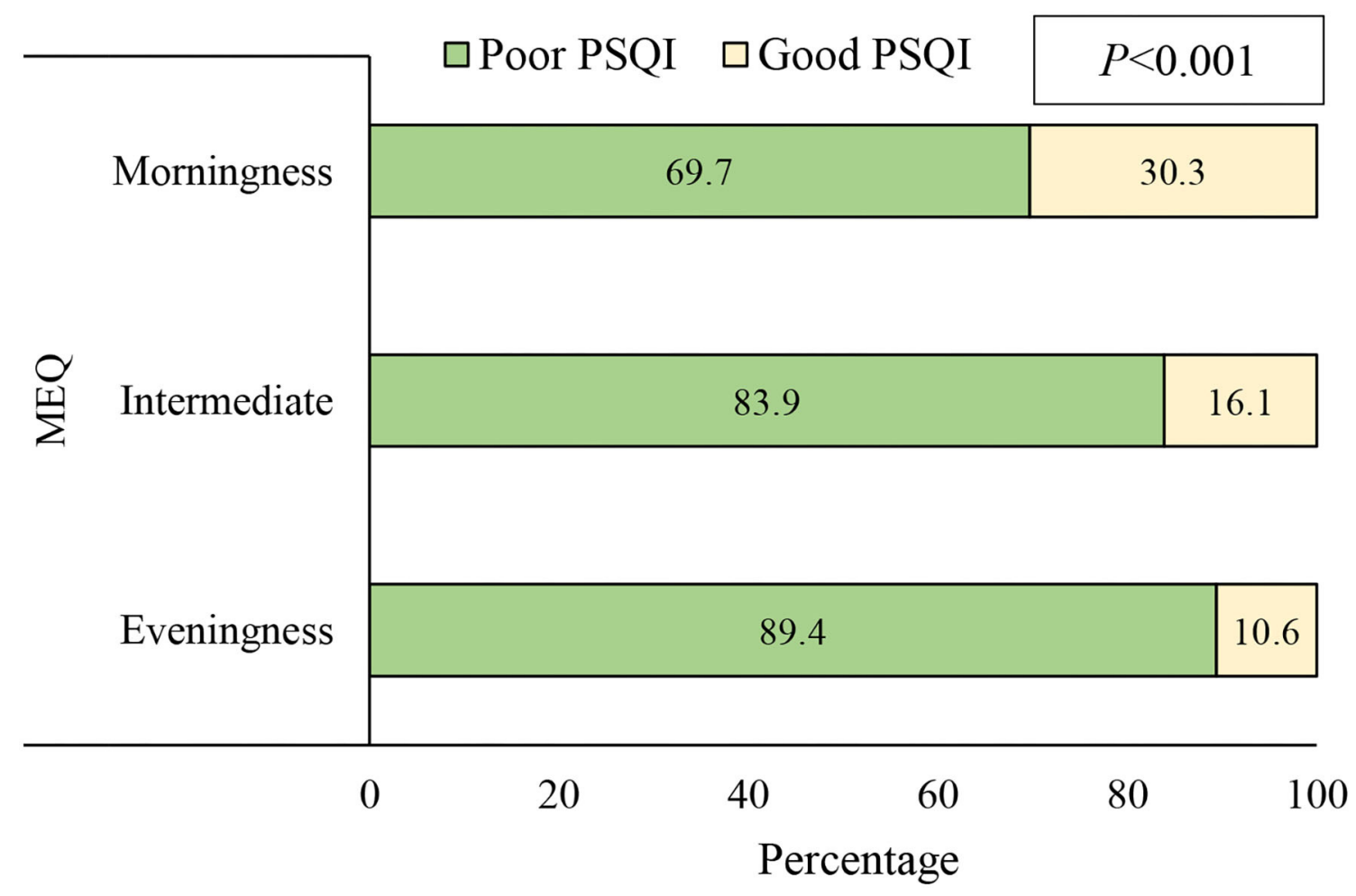

FIGURE 2 | Association of the chronotype, as assessed by Morningness-Eveningness Questionnaire (MEQ), with sleep quality examined using Pittsburgh Sleep Quality Index (PSQI) among study participants $(n=503)$.

and lower-quality diet, which are increasingly recognized as risk factors for obesity $(58,59)$. It was proposed that short sleep duration affects energy expenditure and/or energy intake in a way that creates a positive energy balance. Indeed, the sleep restriction triggers energy intake that exceeds the added energy costs of maintaining longer wakefulness (58).

From a health perspective, the morning chronotype has been the preferred chronotype over the evening one, with the former having less association with cardiometabolic risk factors and other health problems, including psychological, neurological, and gastrointestinal morbidities, and lower mortality rates compared to evening chronotypes (69). These health improvements associated with the morning chronotype could be also a mirror for the improved eating habits consistently reported with such chronotype, as revealed by the increased adherence to the MD by people with morning chronotype $(70,71)$. The reported low prevalence of morningness chronotype in comparison with the eveningness one $(15.1 \%$ vs. $24.5 \%$, respectively) among the study sample is expected, considering the frequently observed long stay up at night among surrounding and neighboring students, and in line with the low prevalence reported in other parts of the world. Similar distributions of morning chronotype (15.8 and 18.4\%) were found among university students in Italy and China, respectively (72, 73). Such pattern of chronobiology among university students could be explained, in part, by the same aforementioned factors affecting their food selection and eating behaviors; that is the hectic schedule, high demanding and frequent academic tasks, and exams, with the students' persistent endeavor to catch the due dates and deadlines and to find less distracting times and more silent surrounding environment. Further, the excessive use of mobile applications and the massive reliance on blue screens for socialization play a role, in part, for such outcomes. Thirdly, the reported positive correlation between eveningness and higher cognitive ability [explaining why more people with higher cognitive ability are more likely to be nocturnal than others (74)] may explain the predominance of eveningness chronotype over other chronotypes among university students (73). Lastly, the frequently reported consumption of stimulant beverages such as caffeine-rich energy drinks and coffees $(32,33,75)$ is among the fundamental factors for extending the wake night hours and shifting the chronotype into the eveningness pattern among university students. This finding of the predominance of eveningness pattern is consistent with the increased prevalence of sleep problems among university students, especially medical students, as revealed by our previously published systematic review and meta-analysis (39).

The relatively low prevalence of good sleep quality (about $17 \%$ ) among our university students is also consistent with and mirrors for other reports in the UAE and other parts of the globe. However, this number is lower than previous estimates among students in the UAE, whereby the prevalence rates of good sleep, using similar assessment tools were $34 \%$ in 2020 and $44.8 \%$ in 2021 , respectively $(50,76)$. Such a high heterogeneity in the 
TABLE 3 | Simple and multiple logistic regression for the association of sleep quality with adherence to the Mediterranean diet among study participants $(n=503)$.

\begin{tabular}{|c|c|c|c|c|c|c|c|c|c|c|c|}
\hline & & \multirow[t]{2}{*}{$B$} & \multicolumn{4}{|c|}{ Unadjusted } & \multirow[t]{2}{*}{$B$} & \multicolumn{4}{|c|}{ Adjusted $^{*}$} \\
\hline & & & OR & Lower & Upper & $P$-value & & OR & Lower & Upper & $P$-value \\
\hline \multicolumn{12}{|c|}{ Overall PSQI** } \\
\hline KIDMED*** & Low & Ref. & - & - & - & - & Ref. & - & - & - & - \\
\hline \multirow[t]{2}{*}{ KIDMED } & Low & Ref. & - & - & - & - & Ref. & - & - & - & - \\
\hline & High & -0.78 & 0.46 & 0.31 & 0.67 & $<0.001$ & -0.69 & 0.50 & 0.34 & 0.73 & $<0.001$ \\
\hline \multicolumn{12}{|c|}{ Sleep latency ${ }^{\star *}$} \\
\hline \multirow[t]{2}{*}{ KIDMED } & Low & Ref. & - & - & - & - & Ref. & - & - & - & - \\
\hline & High & -0.53 & 0.58 & 0.41 & 0.83 & 0.003 & -0.55 & 0.58 & 0.40 & 0.83 & 0.003 \\
\hline \multirow[t]{2}{*}{ KIDMED } & Low & Ref. & - & - & - & - & Ref. & - & - & - & - \\
\hline & High & -0.21 & 0.81 & 0.55 & 1.19 & 0.29 & -0.20 & 0.81 & 0.54 & 1.20 & 0.30 \\
\hline \multicolumn{12}{|c|}{ Sleep disturbance ${ }^{\star \star}$} \\
\hline \multirow[t]{2}{*}{ KIDMED } & Low & Ref. & - & - & - & - & Ref. & - & - & - & - \\
\hline & High & -0.39 & 0.67 & 0.46 & 0.98 & 0.04 & -0.37 & 0.69 & 0.47 & 1.00 & 0.05 \\
\hline \multicolumn{12}{|c|}{ Use of sleep medication ${ }^{\star *}$} \\
\hline \multirow[t]{2}{*}{ KIDMED } & Low & Ref. & - & - & - & - & Ref. & - & - & - & - \\
\hline & High & -0.09 & 0.90 & 0.51 & 1.16 & 0.74 & -0.08 & 0.91 & 0.51 & 1.64 & 0.77 \\
\hline \multicolumn{12}{|c|}{ Daytime dysfunction ${ }^{\star *}$} \\
\hline KIDMED & Low & Ref. & - & - & - & - & Ref. & - & - & - & - \\
\hline
\end{tabular}

PSQI, Pittsburgh Sleep Quality Index; Cl, Confidence Interval.

*Adjusted for age and sex.

${ }^{* *}$ Dependent variable.

${ }^{* * *}$ Independent variable.

Low is poor adherence to KIDMED and high indicates average and good adherence to KIDMED.

reported prevalence of sleep quality among students in the same institute during two consecutive years is a mirror for the wide spectrum of interacting and interplaying variables impacting sleep quality, and the high sensitivity of sleep quality for variable environmental (dietary and lifestyle) factors. Parallel with the previous outcomes, the use of energy drinks among university students in the UoS was found to be significantly associated with reduced sleep quality, as revealed in our previous work in 2017 (32).

Having three-times higher reported good sleep quality among those with morningness chronotype than those with the evening one $(30.3 \%$ vs. $10.6 \%)$, found in this study, is consistent with other reports among university students throughout the world, such as in Brazil (77, 78), India (79) and Korea (80), where eveningness chronotype was consistently associated with poorer sleep quality. Such a strong relationship is a reflection of the direct interaction between circadian rhythm and sleep in response to the chronotype (81). In a similar work among university students in Brazil, evening-style students with poor sleep quality presented shorter total sleep time, higher sleep latency, and late-onset of sleep; all imply a kind of lack of compatibility between the biological rhythms and the morning academic demands. Such incompatibility generates successive daily desynchronizations. Further, with morning academic duties, students with evening chronotype are more prone to be anxious in relation to their personality, which indicates a greater irregularity of the sleep-wake cycle than the other chronotypes, and puts them at higher risk of impairment of cognitive and psychological processes, social jet lag, and consequently, worse quality of life and academic performance and (78).

Age and sex are factors that are variably associated with the adherence to the MD (60), with conflicting results that have been found for the relationships with adherence to MD (61). Consistent with our findings, older age is one of the determining factors for the degree of adherence to the $\operatorname{MD}(60,62)$. For instance, lower adherence to the MD during two decades from 1985 to 2005 in South Italy was found to be more pronounced in younger people than in older people (30-49 vs. 50-69 years) (62), while higher age was also associated with more adherence to the MD among patients with food neophobia in Italy (60). These 
TABLE 4 | Logistic regression for the association of KIDMED with the chronotype among study participants $(n=503)$.

\begin{tabular}{|c|c|c|c|c|c|}
\hline \multirow[t]{3}{*}{ Model } & \multirow[t]{3}{*}{$\begin{array}{l}\text { Independent } \\
\text { variables }\end{array}$} & \multirow[t]{3}{*}{ OR } & \multirow{2}{*}{\multicolumn{2}{|c|}{$\begin{array}{c}\text { KIDMED (Dependent } \\
\text { variable) }\end{array}$}} & \multirow[t]{3}{*}{$p$-value } \\
\hline & & & & & \\
\hline & & & Lower & Upper & \\
\hline \multirow[t]{4}{*}{ Unadjusted } & MEQ & & & & \\
\hline & Eveningness & Ref & & & \\
\hline & Intermediate & 1.72 & 1.13 & 2.64 & 0.012 \\
\hline & Morningness & 6.14 & 3.14 & 12.00 & $<0.001$ \\
\hline \multirow[t]{6}{*}{ Adjusted } & MEQ & & & & \\
\hline & Eveningness & Ref & & & \\
\hline & Intermediate & 1.64 & 1.07 & 2.54 & 0.024 \\
\hline & Morningness & 5.67 & 2.86 & 11.26 & $<0.001$ \\
\hline & Age & 1.05 & 1.00 & 1.11 & 0.035 \\
\hline & Sex & 0.51 & 0.31 & 0.84 & 0.008 \\
\hline
\end{tabular}

MEQ, Morningness-Eveningness Questionnaire; Cl, Confidence Interval.

findings are in line with the dominant nutrition transition taking place in many countries in the world, where traditional and healthier dietary patterns are slowly eroding and being replaced by "westernized" patterns of food consumption. This nutrition transition is most pronounced among the younger population, who are most susceptible to the globalization of food intake and the influence of fast food and processed food chains $(63,64)$.

Despite the well-known health-improving effects and diseasepreventing impact of MD against chronic diseases, a low adherence rate to the MD has been repeatedly reported, especially in the Mediterranean countries themselves $(61,65)$, with sex has been identified as one of the multiple factors working as predictors of poor adherence to the MD (61). The superiority of females in reporting more adherence to the MD diet in our work is consistent with previous work showing that male personality traits were associated with low MD adherence among patients with ischemic heart disease (IHD) (66). However, female sex was shown to be more associated with low adherence in comparison to males in another study in Italy among adults with IHD (61), implying that various other factors such as age, health condition, cultural, demographic, and socioeconomic variables may play a role in determining the level of adherence to the MD.

Having fewer fruits than recommended, skipping breakfast, and frequently visiting fast-food restaurants reported among university students in our work is consistent with the repeatedly observed unhealthy behaviors among the university students in the UAE and other parts of the world $(67,68)$. University students' diets are low in fruits and vegetables and high in fats, carbohydrates, and sodium, making them energy-dense yet nutrient-poor options. In the UAE, university students have been found to eat unplanned meals consisting primarily of quick, unhealthy, energy-dense foods (36), and have been observed to consume a lot of fast food and soft drinks, as well as skipping meals $(34,37)$. Such unhealthy eating behaviors and poor food selections by university students have been ascribed to variable environmental factors that characterize the university academic life, such as the hectic schedule, high demanding and frequent academic tasks, and exams.

The current work entails some limitations that should be considered when interpreting the current findings. First, diet quantity was not examined in the current work, which may also affect sleep quality and may interact with different components of sleep. The inherent limitations of the cross-sectional design cannot be overridden in this context, where causality cannot be inferred in such an observational design. Other limitations related to the self-reported questionnaires such as recall bias, and response bias are worth to be kept in mind. Of particular importance is the self-reported nature of the assessment of sleep quality, which relies heavily on the participants' subjective assessment. Future studies could include objective measures of sleep quality, using polysomnography (82). In addition, this study explored only the associations between adherence to MD and sleep quality, not the mechanisms behind these associations. This study also does not account for possible confounding factors that may affect the relationship, such as evening thermogenic factors like nocturnal exercise, nocturnal light exposure, and spicy nocturnal meals, and physical activity level. Lastly, adjustments for the alpha value for multiple comparisons that were not performed need to be considered in future studies.

To conclude, good adherence to the MD among university students is more associated with morningness chronotype and improved overall sleep quality and sleep components. Improving the knowledge and attitude of the university students toward their dietary and lifestyle behaviors, and the significance of chronotype in determining their future disease risk factors are of pivotal importance. Further long-term, controlled intervention research works are warranted for more elaboration on the impact of chronotype and dietary habits on sleep quality.

\section{DATA AVAILABILITY STATEMENT}

The raw data supporting the conclusions of this article will be made available by the authors, without undue reservation.

\section{ETHICS STATEMENT}

The studies involving human participants were reviewed and approved by University of Sharjah, REC approval no. (REC21-03-03-09-S). The patients/participants provided their written informed consent to participate in this study.

\section{AUTHOR CONTRIBUTIONS}

MF: conceptualization, project administration, and funding acquisition. MF and FN: methodology, validation, supervision, and writing-original draft. $\mathrm{HH}$ : software. $\mathrm{HH}$ and $\mathrm{FN}$ : formal analysis and visualization. $\mathrm{MF}, \mathrm{FN}, \mathrm{SK}, \mathrm{MB}$, HA-M, and AA: investigation and data curation. MF, $\mathrm{FN}$, and $\mathrm{HH}$ : resources and writing-review and editing. All authors contributed to the article and approved the submitted version. 


\section{REFERENCES}

1. Dernini S, Berry EM, Serra-Majem L, La Vecchia C, Capone R, Medina F, et al. Med Diet 4.0: the Mediterranean diet with four sustainable benefits. Public Health Nutr. (2017) 20:1322-30. doi: 10.1017/S1368980016003177

2. Martinez-Lacoba R, Pardo-Garcia I, Amo-Saus E, Escribano-Sotos F. Mediterranean diet and health outcomes: a systematic meta-review. Eur J Public Health. (2018) 28:955-61. doi: 10.1093/eurpub/cky113

3. El Hajj JS, Julien SG. Factors associated with adherence to the mediterranean diet and dietary habits among University Students in Lebanon. J Nutr Metab. (2021) 2021:6688462. doi: 10.1155/2021/6688462

4. de la Torre-Moral A, Fàbregues S, Bach-Faig A, Fornieles-Deu A, Medina FX, Aguilar-Martínez A, et al. Family meals, conviviality, and the mediterranean diet among families with adolescents. Int J Environ Res Public Health. (2021) 18:2499. doi: 10.3390/ijerph18052499

5. Martínez-González MA, Salas-Salvadó J, Estruch R, Corella D, Fitó M, Ros E. et al. Benefits of the Mediterranean diet: insights from the PREDIMED study. Progr Cardiovasc Dis. (2015) 58:50-60. doi: 10.1016/j.pcad. 2015.04.003

6. Nagpal R, Shively CA, Register TC, Craft S, Yadav H. Gut microbiomeMediterranean diet interactions in improving host health. F1000Res. (2019) 8:699. doi: 10.12688/f1000research.18992.1

7. Schwingshackl L, Chaimani A, Hoffmann G, Schwedhelm C, Boeing H. A network meta-analysis on the comparative efficacy of different dietary approaches on glycaemic control in patients with type 2 diabetes mellitus. Eur J Epidemiol. (2018) 33:157-70. doi: 10.1007/ s10654-017-0352-x

8. Becerra-Tomás N, Blanco Mejía S, Viguiliouk E, Khan T, Kendall CW, Kahleova H. et al. Mediterranean diet, cardiovascular disease and mortality in diabetes: A systematic review and meta-analysis of prospective cohort studies and randomized clinical trials. Crit Rev Food Sci Nutr. (2020) 60:120727. doi: 10.1080/10408398.2019.1565281

9. G. Marrazzo*, I. Barbagallo*, F. Galvano, M. Malaguarnera, D. Gazzolo, A. Frigiola, et al. Role of dietary and endogenous antioxidants in diabetes. Crit Rev Food Sci Nutr. (2014) 54:1599-616. doi: 10.1080/10408398.2011.644874

10. Paniagua JA, de la Sacristana AG, Sánchez E, Romero I, Vidal-Puig A, Berral FJ, et al. A MUFA-rich diet improves posprandial glucose, lipid and GLP1 responses in insulin-resistant subjects. J erican Coll Nutr. (2007) 26:43444. doi: 10.1080/07315724.2007.10719633

11. Casas R, Sacanella E, Estruch R. The immune protective effect of the Mediterranean diet against chronic low-grade inflammatory diseases. Endocr Metab Immune Disord Drug Targets. (2014) 14:245-54. doi: 10.2174/1871530314666140922153350

12. Simpson N, Dinges DF. Sleep and inflammation. Nutr Rev. (2007) 65:S24452. doi: 10.1111/j.1753-4887.2007.tb00371.x

13. Milrad SF, Hall DL, Jutagir DR, Lattie EG, Ironson GH, Wohlgemuth W. et al. Poor sleep quality is associated with greater circulating pro-inflammatory cytokines and severity and frequency of chronic fatigue syndrome/myalgic encephalomyelitis (CFS/ME) symptoms in women. J Neuroimmunol. (2017) 303:43-50. doi: 10.1016/j.jneuroim.2016.12.008

14. Goel N, Basner M, Rao H, Dinges DF. Circadian rhythms, sleep deprivation, and human performance. Prog Mol Biol Transl Sci. (2013) 119:15590. doi: 10.1016/B978-0-12-396971-2.00007-5

15. Schlarb AA, Friedrich A, Claßen M. Sleep problems in university students-an intervention. Neuropsychiatr Dis Treat. (2017) 13:1989. doi: 10.2147/NDT.S142067

16. Chattu VK, Manzar MD, Kumary S, Burman D, Spence DW, Pandi-Perumal SR. The global problem of insufficient sleep and its serious public health implications. Healthcare. (2018) 7:1. doi: 10.3390/healthcare7010001

17. Rafique N, Al-Asoom LI, Alsunni AA, Saudagar FN, Almulhim L, Alkaltham G. Effects of Mobile Use on Subjective Sleep Quality. Nat Sci Sleep. (2020) 12:357-64. doi: 10.2147/NSS.S253375

18. Javaheri S, Omobomi O, Redline S. Insufficient Sleep and Cardiovascular Disease Risk, Sleep and Health. Netherlands:Elsevier (2019), p. 20312. doi: 10.1016/B978-0-12-815373-4.00016-2

19. Adan SN, Archer MP, Hidalgo L, Di Milia, Natale V, Randler C. Circadian typology: a comprehensive review. Chronobiol Int. (2012) 29:115375. doi: 10.3109/07420528.2012.719971
20. Duarte L, Menna-Barreto L, Miguel M, Louzada F, Araújo J, Alam M. et al. Chronotype ontogeny related to gender. Braz J Med Biol Res. (2014) 47:316-20. doi: 10.1590/1414-431X20143001

21. Leocadio-Miguel MA, Louzada FM, Duarte LL, Areas RP, Alam M, Freire MV. et al. Latitudinal cline of chronotype. Sci Rep. (2017) 7:16. doi: 10.1038/s41598-017-05797-w

22. Juda M, Vetter C, Roenneberg T. Chronotype modulates sleep duration, sleep quality, and social jet lag in shift-workers. J Biol Rhythms. (2013) 28:141-51. doi: 10.1177/0748730412475042

23. Martínez-Lozano N, Barraco GM, Rios R, Ruiz MJ, Tvarijonaviciute A, Fardy P. et al. Evening types have social jet lag and metabolic alterations in schoolage children. Sci Rep. (2020) 10:1-11. doi: 10.1038/s41598-020-73297-5

24. Taillard J, Sagaspe P, Philip P, Bioulac S. Sleep timing, chronotype and social jetlag: Impact on cognitive abilities and psychiatric disorders. Biochem Pharmacol. (2021) 191:114438. doi: 10.1016/j.bcp.2021.114438

25. Binks H, Vincent GE, Gupta C, Irwin C, Khalesi S. Effects of diet on sleep: a narrative review. Nutrients. (2020) 12:936. doi: 10.3390/nu12040936

26. Zuraikat FM, Wood RA, Barragán R, St-Onge M-P. Sleep and diet: mounting evidence of a cyclical relationship. Annu Rev Nutr. (2021) 41:30932. doi: 10.1146/annurev-nutr-120420-021719

27. Peuhkuri K, Sihvola N, Korpela R. Diet promotes sleep duration and quality. Nutr Res. (2012) 32:309-19. doi: 10.1016/j.nutres.2012.03.009

28. Zhao M, Tuo H, Wang S, Zhao L. The effects of dietary nutrition on sleep and sleep disorders. Mediators Inflamm. (2020) 2020:3142874. doi: 10.1155/2020/3142874

29. Wood D, Crapnell T, Lau L, Bennett A, Lotstein D, Ferris M, et al. Emerging Adulthood as a Critical Stage in the Life Course. Berlin: Springer International Publishing (2018), pp. 123-43. doi: 10.1007/978-3-319-47143-3_7

30. Arnett JJ, Kloep M, Hendry LB, Tanner JL. Debating Emerging Adulthood: Stage or Process? UK: Oxford University Press (2011). doi: 10.1093/acprof:oso/9780199757176.001.0001

31. Welle PD, Graf HM. Effective lifestyle habits and coping strategies for stress tolerance among college students. Am J Health Educ. (2011) 42:96105. doi: 10.1080/19325037.2011.10599177

32. Faris ME, Jahrami H, AlHilali MM, Chehyber NJ, Ali SO, Shahda SD. et al. Energy drink consumption is associated with reduced sleep quality among college students: a cross-sectional study. Nutr Diet. (2017) 74:26874. doi: 10.1111/1747-0080.12289

33. Jahrami H, Al-Mutarid M, Penson PE, Faris ME, Saif Z, Hammad L. Intake of caffeine and its association with physical and mental health status among university students in Bahrain. Foods. (2020) 9:473. doi: 10.3390/foods 9040473

34. Kerkadi. Evaluation of nutritional status of United Arab Emirates University female students. Emirates J Food Agri. (2003) 42-50. doi: 10.9755/ejfa.v15i2.5005

35. Tey SL, Brown RC, Gray AR, Chisholm AW, Delahunty CM. Long-term consumption of high energy-dense snack foods on sensory-specific satiety and intake. Am J Clin Nutr. (2012) 95:1038-47. doi: 10.3945/ajcn.111.030882

36. Musaiger AO, Lloyd OL, Al?Neyadi SM, Bener AB. Lifestyle factors associated with obesity among male university students in the United Arab Emirates. Nutr Food Sci. (2003) 33:145-7. doi: 10.1108/00346650310488480

37. Mahmood M, Saleh A, Al-Alawi F, Ahmed F. Health effects of soda drinking in adolescent girls in the United Arab Emirates. J Crit Care. (2008) 23:43440. doi: 10.1016/j.jcrc.2008.06.006

38. Peltzer K, Pengpid S. Nocturnal sleep problems among university students from 26 countries. Sleep and Breathing. (2015) 19:499508. doi: 10.1007/s11325-014-1036-3

39. Jahrami H, Dewald-Kaufmann J, Faris ME, AlAnsari AM, Taha M, AlAnsari N. Prevalence of sleep problems among medical students: a systematic review and meta-analysis. J Publ Health. (2019) 1-18. doi: 10.1007/s10389-019-01064-6

40. http://www.raosoft.com/samplesize.html, Sample size calculator (2004).

41. Mitri R, Boulos C, Ziade F. Mediterranean diet adherence among adolescents in North Lebanon: the role of skipping meals, meals with the family, physical activity, and physical wellbeing. Br J Nutr. 127. doi: 10.1017/S0007114521002269

42. Atencio-Osorio MA, Carrillo-Arango HA, Correa-Rodríguez M, OchoaMuñoz AF, Ramírez-Vélez R. Adherence to the mediterranean diet in 
college students: evaluation of psychometric properties of the KIDMED questionnaire. Nutrients. (2020) 12:3897. doi: 10.3390/nu12123897

43. Štefan L, Prosoli R, Juranko D, Cule M, Milinović I, Novak D. et al. The reliability of the mediterranean diet quality index (KIDMED) questionnaire. Nutrients. (2017) 9:419. doi: 10.3390/nu9040419

44. Serra-Majem L, Ribas L, Ngo J, Ortega RM, García A, Pérez-Rodrigo C. et al. Food, youth and the Mediterranean diet in Spain Development of KIDMED, Mediterranean Diet Quality Index in children and adolescents. Publ Health Nutr. (2004) 7:931-5. doi: 10.1079/PHN2004556

45. Buysse DJ, Reynolds III CF, Monk TH, Berman SR, Kupfer DJ. The Pittsburgh Sleep Quality Index: a new instrument for psychiatric practice and research. Psychiatry Res. (1989) 28:193-213. doi: 10.1016/01651781(89)90047-4

46. Suleiman KH, Yates BC, Berger AM, Pozehl B, Meza J. Translating the Pittsburgh Sleep Quality Index Into Arabic. West J Nurs Res. (2010) 32:25068. doi: 10.1177/0193945909348230

47. Horne JA, Östberg O. A self-assessment questionnaire to determine morningness-eveningness in human circadian rhythms. Int $J$ Chronobiol. (1976).

48. Levandovski R, Sasso E, Hidalgo MP. Chronotype: a review of the advances, limits and applicability of the main instruments used in the literature to assess human phenotype. Trends Psychiatry Psychother. (2013) 35:311. doi: $10.1590 /$ S2237-60892013000100002

49. Mansour H, Tobar S, Fathi W, Ibrahim I, Wood J, Elassy M. et al. Arabic versions of the sleep timing questionnaire and the composite scale of morningness. Asian J Psychiatry. (2015) 13:48-51. doi: 10.1016/j.ajp.2014.10.004

50. Masaad AA, Yusuf AM, Shakir AZ, Khan MS, Khaleel S, Ismail LC, et al. Sleep quality and dietary inflammatory index among university students: a cross-sectional study. Sleep Breath. (2020) 1-9.

51. Godos J, Ferri R, Caraci F, Cosentino FII, Castellano S, Shivappa N. et al. Dietary inflammatory index and sleep quality in Southern Italian Adults. Nutrients. (2019) 11:1324. doi: 10.3390/nu11061324

52. Irwin MR, Olmstead R, Carroll JE. Sleep disturbance, sleep duration, and inflammation: a systematic review and meta-analysis of cohort studies and experimental sleep deprivation. Biol Psychiatry. (2016) 80:4052. doi: 10.1016/j.biopsych.2015.05.014

53. Nani, Murtaza B, Sayed Khan A, Khan NA, Hichami A. Antioxidant and anti-inflammatory potential of polyphenols contained in mediterranean diet in obesity: molecular mechanisms. Molecules. (2021) 26:985. doi: 10.3390/molecules 26040985

54. Motivala SJ. Sleep and inflammation: psychoneuroimmunology in the context of cardiovascular disease. Ann Behav Med. (2011) 42:14152. doi: 10.1007/s12160-011-9280-2

55. Mills PJ, von Känel R, Norman D, Natarajan L, Ziegler MG, Dimsdale JE. Inflammation and sleep in healthy individuals. Sleep. (2007) 30:72935. doi: 10.1093/sleep/30.6.729

56. Camargo, Delgado-Lista J, Garcia-Rios A, Cruz-Teno C, Yubero-Serrano EM, Perez-Martinez $\mathrm{P}$, et al. Expression of proinflammatory, proatherogenic genes is reduced by the Mediterranean diet in elderly people. Br J Nutr. (2012) 108:500-8. doi: 10.1017/S0007114511005812

57. Zuraikat FM, Makarem N, St-Onge M-P, Xi H, Akkapeddi A, Aggarwal B. A Mediterranean Dietary Pattern Predicts Better Sleep Quality in US Women from the American Heart Association Go Red for Women Strategically Focused Research Network. Nutrients. (2020) 12:2830. doi: 10.3390/nu12092830

58. Shechter, Grandner MA, St-Onge M-P. The role of sleep in the control of food intake. Am J Lifestyle Med. (2014) 8:371-4. doi: 10.1177/1559827614545315

59. Zuraikat FM, Makarem N, Liao M, St-Onge MP, Aggarwal B. Measures of poor sleep quality are associated with higher energy intake and poor diet quality in a diverse sample of women from the go red for women strategically focused research network. J Am Heart Assoc. (2020) 9:e014587. doi: 10.1161/JAHA.119.014587

60. Predieri S, Sinesio F, Monteleone E, Spinelli S, Cianciabella M, Daniele GM. et al. Gender, age, geographical area, food neophobia and their relationships with the adherence to the mediterranean diet: new insights from a large population cross-sectional study. Nutrients. (2020) 12:1778. doi: 10.3390/nu12061778
61. Downer MK, Gea A, Stampfer M, Sánchez-Tainta A, Corella D, Salas-Salvadó $\mathrm{J}$, et al. Predictors of short- and long-term adherence with a Mediterraneantype diet intervention: the PREDIMED randomized trial. Int J Behav Nutr Phys Act. (2016) 13:67. doi: 10.1186/s12966-016-0394-6

62. Veronese $\mathrm{N}$, Notarnicola $\mathrm{M}$, Cisternino AM, Inguaggiato R, Guerra V, Reddavide R. et al. Trends in adherence to the Mediterranean diet in South Italy: A cross sectional study. Nutr Metab Cardiovasc Dis. (2020) 30:4107. doi: 10.1016/j.numecd.2019.11.003

63. Popkin BM, Adair LS, Ng SW. Global nutrition transition and the pandemic of obesity in developing countries. Nutr Rev. (2012) 70:321. doi: 10.1111/j.1753-4887.2011.00456.x

64. Donders, Barriocanal C. The influence of markets on the nutrition transition of hunter-gatherers: lessons from the Western Amazon. Int J Environ Res Public Health. (2020) 17:6307. doi: 10.3390/ijerph17176307

65. Silva RDa, Bach-Faig A, Quintana BR, Buckland G, Almeida MDVde, Serra-Majem L. Worldwide variation of adherence to the Mediterranean diet, in 1961-1965 and 2000-2003. Public Health Nutr. (2009) 12:167684. doi: 10.1017/S1368980009990541

66. Raparelli V, Romiti GF, Spugnardi V, Borgi M, Cangemi R, Basili S. et al. The eva collaborative, gender-related determinants of adherence to the mediterranean diet in adults with ischemic heart disease. Nutrients. (2020) 12:759. doi: $10.3390 /$ nu12030759

67. Llanaj E, Ádány R, Lachat C, D’Haese M. Examining food intake and eating out of home patterns among university students. PLoS ONE. (2018) 13:e0197874. doi: 10.1371/journal.pone.0197874

68. Sprake EF, Russell JM, Cecil JE, Cooper RJ, Grabowski P, Pourshahidi LK, et al. Dietary patterns of university students in the UK: a cross-sectional study. Nutr J. (2018) 17. doi: 10.1186/s12937-018-0398-y

69. Makarem N, Paul J, Giardina E-GV, Liao M, Aggarwal B. Evening chronotype is associated with poor cardiovascular health and adverse health behaviors in a diverse population of women. Chronobiol Int. (2020) 37:67385. doi: 10.1080/07420528.2020.1732403

70. Muscogiuri G, Barrea L, Aprano S, Framondi L, Matteo RDi, Laudisio D. et al. Chronotype and adherence to the mediterranean diet in obesity: results from the opera prevention project. Nutrients. (2020) 12:1354. doi: $10.3390 /$ nu12051354

71. Rodríguez-Muñoz PM, Carmona-Torres JM, Rivera-Picón C, Fabbian F, Manfredini R, Rodríguez-Borrego MA. et al. Associations between chronotype, adherence to the mediterranean diet and sexual opinion among university students. Nutrients. (2020) 12:1900. doi: 10.3390/ nu12061900

72. Li T, Xie Y, Tao S, Yang Y, Xu H, Zou L, et al. Chronotype, sleep, and depressive symptoms among chinese college students: a crosssectional study. Front Neurol. (2020) 11:592825. doi: 10.3389/fneur. 2020.592825

73. Montaruli, Castelli L, Galasso L, Mulè A, Bruno E, Esposito F, et al. Effect of chronotype on academic achievement in a sample of Italian University students. Chronobiol Int. (2019) 36:1482-95. doi: 10.1080/07420528.2019.1652831

74. Preckel F, Lipnevich AA, Schneider S, Roberts RD. Chronotype, cognitive abilities, and academic achievement: A meta-analytic investigation. Learn Individ Differ. (2011) 21:483-92. doi: 10.1016/j.lindif. 2011.07.003

75. Faris M. Patterns of caffeinated energy drinks consumption among adolescents and adults in hail, Saudi Arabia. Nutr Food Sci. (2014) 5:158-68. doi: 10.4236/fns.2014.52021

76. Faris ME, Vitiello MV, Abdelrahim DN, Cheikh Ismail L, Jahrami HA, Khaleel $\mathrm{S}$, et al. Eating habits are associated with subjective sleep quality outcomes among university students: findings of a cross-sectional study. Sleep Breath. (2021) 1-12.

77. Rique GLN, Fernandes Filho GMC, Ferreira ADC, Sousa-Muñoz RLDe. Relationship between chronotype and quality of sleep in medical students at the Federal University of Paraiba, Brazil. Sleep Sci. (2014) 7:96102. doi: 10.1016/j.slsci.2014.09.004

78. Silva VM, Magalhaes JEDM, Duarte LL. Quality of sleep and anxiety are related to circadian preference in university students. PLoS ONE. (2020) 15:e238514. doi: 10.1371/journal.pone.0238514 
79. Gangwar A, Tiwari S, Rawat A, Verma A, Singh K, Kant S, et al. Circadian preference, sleep quality, and health-impairing lifestyles among undergraduates of medical university. Cureus. (2018) 10:e2856. doi: 10.7759/cureus.2856

80. Yun J-A, Ahn Y-S, Jeong K-S, Joo E-J, Choi K-S. The Relationship between Chronotype and Sleep Quality in Korean Firefighters. Clin Psychopharmacol Neurosci. (2015) 13:201-8. doi: 10.9758/cpn.2015.13.2.201

81. Vitale JA, Roveda E, Montaruli A, Galasso L, Weydahl A, Caumo A. et al. Chronotype influences activity circadian rhythm and sleep: differences in sleep quality between weekdays and weekend. Chronobiol Int. (2015) 32:405-15. doi: 10.3109/07420528.2014. 986273

82. O’Donnell D, Silva EJ, Münch M, Ronda JM, Wang W, Duffy JF. Comparison of subjective and objective assessments of sleep in healthy older subjects without sleep complaints. J Sleep Res. (2009) 18:254-63. doi: 10.1111/j.1365-2869. 2008.00719.x
Conflict of Interest: The authors declare that the research was conducted in the absence of any commercial or financial relationships that could be construed as a potential conflict of interest.

Publisher's Note: All claims expressed in this article are solely those of the authors and do not necessarily represent those of their affiliated organizations, or those of the publisher, the editors and the reviewers. Any product that may be evaluated in this article, or claim that may be made by its manufacturer, is not guaranteed or endorsed by the publisher.

Copyright (c) 2022 Naja, Hasan, Khadem, Buanq, Al-Mulla, Aljassmi and Faris. This is an open-access article distributed under the terms of the Creative Commons Attribution License (CC BY). The use, distribution or reproduction in other forums is permitted, provided the original author(s) and the copyright owner(s) are credited and that the original publication in this journal is cited, in accordance with accepted academic practice. No use, distribution or reproduction is permitted which does not comply with these terms. 